\title{
Increased embolic risk from combined oral contraceptives
}

Women who use oral contraceptives containing estrogen and a progestogen are at a threefold to sixfold higher risk of venous thromboembolism than nonusers of hormonal contraception. This finding comes from a large, retrospective study of all nonpregnant women aged 15-49 years in Denmark between January 2001 and December 2009, which has now been published in the BMJ.

The investigators were commissioned by the European Medicines Agency to conduct this research. Data on $1,296,120$ women were included in the study. A total of 4,307 first-time venous thromboembolic events were recorded, and 2,847 were subsequently confirmed. Compared with women not using hormonal contraception, the relative risk of venous thromboembolism with contraceptives that included 30-40 $\mu \mathrm{g}$ ethinylestradiol and either levonorgestrel, desogestrel, gestodene, or drospirenone was 2.9 (95\% CI 2.2-3.8), 6.6 (95\% CI 5.6-7.8), 6.2 (95\% CI 5.6-7.0), or 6.4 (95\% CI 5.4-7.5), respectively. The risk of confirmed venous thromboembolism was not increased by the use of progesteroneonly contraception, or hormone-releasing intrauterine devices.

The researchers estimate that, to prevent one event of venous thromboembolism in 1 year, approximately 2,000 women would need to change from using oral contraception with desogestrel, gestodene, or drospirenone to one that contains levonorgestrel.

Gregory B. Lim

Original article Lidegaard, $\emptyset$. et al. Risk of venous thromboembolism from use of oral contraceptives containing different progestogens and oestrogen doses: Danish cohort study, 2001-2009. BMJ 343, d6423 (2011) 